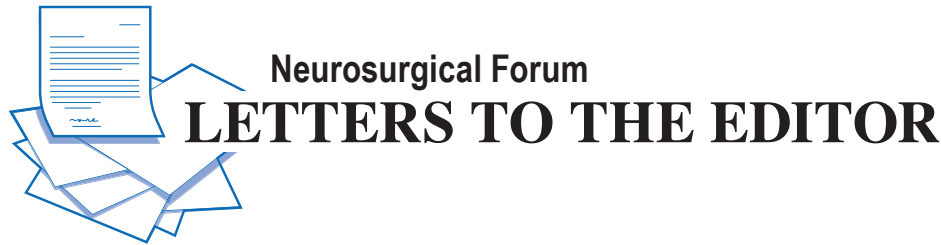

\section{Misinterpretations in the citation}

TO THE EDITOR: I read with interest the article by $\mathrm{Xu}$ et al. ${ }^{2}$ (Xu X, Chen X, Li F, et al: Effectiveness of endoscopic surgery for supratentorial hypertensive intracerebral hemorrhage: a comparison with craniotomy. $J$ Neurosurg [epub ahead of print April 7, 2017. DOI: 10.3171/2016.10.JNS161589]). It is with pleasure that I see my previous work ${ }^{1}$ cited by $\mathrm{Xu}$ and colleagues. Unfortunately, I regret to inform you that aspects of my work are misinterpreted in their paper.

In the paper by $\mathrm{Xu}$ et al., the authors state that there were 20 patients in our study and that these patients had cerebellar hemorrhage. The conclusion of our study is stated as "no improvement in hematoma removal.".

I regret to say that this is not correct. Our study focused on 7 patients who underwent endoscopic evacuation of a posterolateral type of thalamic hemorrhage, which had ruptured into the trigone of the lateral ventricle and caused acute hydrocephalus. In our Conclusion ${ }^{1}$ we stated the following:

Use of a polypropylene endoscopic sheath in combination with an endoscope and an approach from Keen's point to the collateral trigone of the lateral ventricle can improve the efficiency of thalamic hematoma evacuation and prevent shuntdependent hydrocephalus.

Chun-Chung Chen, MD China Medical University Hospital, Taichung, Taiwan

\section{References}

1. Chen CC, Lin HL, Cho DY: Endoscopic surgery for thalamic hemorrhage: a technical note. Surg Neurol 68:438-442, 2007

2. Xu X, Chen X, Li F, Zheng X, Wang Q, Sun G, et al: Effectiveness of endoscopic surgery for supratentorial hypertensive intracerebral hemorrhage: a comparison with craniotomy. J Neurosurg [epub ahead of print April 7, 2017. DOI: 10.3171/2016.10.JNS161589]

\section{Response}

We are really sorry that in Table 3 of our article, we misrepresented the article by Chen et al. Professor Chen and his colleagues reported a case series study involving 7 patients who underwent endoscopic evacuation of tha- lamic hemorrhage that had ruptured into the trigone of the lateral ventricle and caused acute hydrocephalus. The authors' finding in that paper was that endoscopic evacuation improved thalamic hematoma evacuation and prevented shunt-dependent hydrocephalus.

In response to Dr. Chen's Letter to the Editor, we have made appropriate corrections to Table 3 , as outlined in a corresponding erratum notice. We apologize to Prof. Chen and to the Editor and readers of the Journal of Neurosurgery for causing so much trouble and inconvenience. We would like to express our appreciation to Prof. Chen for kindly pointing out our error.

\footnotetext{
Xinghua Xu, MD

Xiaolei Chen, MD Fangye Li, MD

Xuan Zheng, MD

Qun Wang, MD

Guochen Sun, MD

Jun Zhang, MD

Bainan Xu, MD
}

Chinese PLA General Hospital, Beijing, China

\section{INCLUDE WHEN CITING}

Published online July 28, 2017; DOI: 10.3171/2017.4.JNS17909.

CAANS 2018, except where prohibited by US copyright law

\section{Computed tomography angiography in brain death determination: is there justification for its use?}

TO THE EDITOR: We noted the study by Garrett et al., ${ }^{2}$ in which the authors evaluated the utility of CT angiography (CTA) in brain death (BD) (Garrett MP, Williamson RW, Bohl MA, et al: Computed tomography angiography as a confirmatory test for the diagnosis of brain death. J Neurosurg [epub ahead of print March 17, 2017. DOI: 10.3171/2016.10.JNS161042]). CTA has been a research focus in Europe for several years, with both France and Germany incorporating CTA in their BD guidelines, more for convenience (availability) reasons than driven by incontrovertible scientific facts. ${ }^{1}$ 
We would like to comment on several aspects of the recently published study by Garrett et al., which was completed nearly 5 years ago.

First, although the authors are arguably correct for using conventional angiography as the gold standard ancillary test for this study, different criteria were used for CTA and cerebral angiography for the determination of BD. The simplified 4-point criteria used by Frampas et al. ${ }^{1}$ are insufficient, as they do not evaluate for flow in the intracranial internal carotid arteries or in the posterior circulation whatsoever. Conventional angiography evaluates for arrest of flow at the point of entry of all arteries intradurally, both in the anterior and posterior circulations. Why would CTA not be held to the same standard? Evaluation of the posterior circulation is of particular importance in this assessment.

Second, their cerebral angiography criteria for no flow beyond the petrous segment of the carotid artery are debatable because others may claim that cavernous segment filling is allowed, as both are still extradural. If anything, it illustrates the difficulty in defining lack of intracranial flow.

Third, all of the patients in the study underwent clinical determination of BD, but some could not undergo the complete clinical evaluation due to an inability to complete apnea testing or the presence of other confounders. The authors begin with 22 patients in the study, but, in actuality, only 18 patients had a complete "formal diagnostic workup for BD." Of these 18 patients, only 16 actually met the clinical criteria for BD. Thus, it appears that only 16 patients actually met the criteria for enrollment in the study. However, it further turns out that, of these 16 patients, 9 could not undergo complete clinical testing. Thus, in truth, only 7 patients were actually clinically brain dead and underwent subsequent conventional angiography and CTA. The clinical examination cannot be said to be one of the gold standards for this study based on only 7 patients fulfilling the criteria. (Furthermore, 1 patient who underwent CTA experienced cardiac arrest prior to undergoing conventional angiography, and we do not know the details of that case, which may further reduce the study's numbers.) Moreover, how do the clinicians interpret their finding that 3 of 9 cerebral angiogram studies with no flow actually did show flow on CTA? Is this not indicative of tremendous variability in technique?

Putting this aside, the authors proceed to put forth findings that they had $100 \%$ specificity and $75 \%$ sensitivity for BD using CTA, but they do not present confidence intervals or $p$ values. This is because the findings are not statistically significant, and the methods are not sound. The findings would be even more concerning if the total number of appropriate patients included in the study was only 7 , as illustrated above.

It is important to keep in mind important differences between conventional angiography and CTA. Conventional angiography for BD utilizes a power injection, allowing for good visualization of any intracranial arterial flow under pressure. Conversely, CTA is a venous injection, not under pressure, and thus the timing of the contrast bolus to the brain, especially under circumstances of elevated intracranial pressure, might be quite delayed. Conventional angiography is dynamic imaging; CTA is relatively static, and, if the contrast bolus is not timed correctly, intracranial filling could be missed. This especially could be the case in patients with depressed cardiac output, which is quite often the case with brain-dead patients. ${ }^{3,4}$ We do note that the authors built in a 60 -second delay after contrast injection before scanning, which seems reasonable, but, to our knowledge, this is not a validated or universally accepted standard. More broadly, even the imaging reference standard of catheter angiography is subject to variability, as the extent of skull base and intracranial arterial opacification can depend much on the catheter tip position relative to the carotid bifurcation and vertebral artery origins, presence or absence of carotid or vertebral artery stenotic disease, and contrast material injection rates and volumes.

Finally, the single neuroradiologist was not blinded to the clinical data or the findings of cerebral angiography, introducing a significant bias. A more rigorous study should include blinded evaluations of all imaging studies and multiple reviewers with appropriate clinical expertise.

As the authors note, false positives with CTA have been reported, and, based on the findings from these authors, we should not have an increased sense of security in the value of CTA in $\mathrm{BD}$. The possibility of false positives remains quite real, and, without adequate study, CTA should not be utilized in the evaluation of BD as an ancillary test. We would be very concerned if this paper were to lead to incorporating CTA in BD determination in US practice, despite the authors' repeated claims that their decisions are based on a clinical examination. In fact, they are not based on clinical examination, since the authors used cerebral angiography or CTA in patients with confounders ("10 positive urine toxicology"). The broader point is that, for neurosurgeons, this paper is not a justifying reason to use CTA, and there is no "compelling argument" to include CTA in future national guideline revisions. ${ }^{5}$

David M. Greer, MD, MA

Boston University, Boston, MA

Eelco F. M. Wijdicks, MD, PhD

Timothy J. Kaufmann, MD, MS

Mayo Clinic, Rochester, MN

\section{References}

1. Frampas E, Videcoq M, de Kerviler E, Ricolfi F, Kuoch V, Mourey F, et al: CT angiography for brain death diagnosis. AJNR Am J Neuroradiol 30:1566-1570, 2009

2. Garrett MP, Williamson RW, Bohl MA, Bird CR, Theodore $\mathrm{N}$ : Computed tomography angiography as a confirmatory test for the diagnosis of brain death. J Neurosurg [epub ahead of print March 17, 2017. DOI: 10.3171/2016.10.JNS161042]

3. Greer DM, Strozyk D, Schwamm LH: False positive CT angiography in brain death. Neurocrit Care 11:272-275, 2009

4. Wijdicks EFM: The case against confirmatory tests for determining brain death in adults. Neurology 75:77-83, 2010

5. Wijdicks EFM, Varelas PN, Gronseth GS, Greer DM:

Evidence-based guideline update: determining brain death in adults: report of the Quality Standards Subcommittee of the American Academy of Neurology. Neurology 74:1911-1918, 2010

\section{Disclosures}

The authors report no conflict of interest. 


\section{Response}

We appreciate the interest and comments of Drs. Greer, Wijdicks, and Kaufmann regarding the justification and use of CTA as an ancillary test for determination of BD, particularly in comparison with cerebral angiography findings.

Despite cerebral angiography being the largely accepted gold standard ancillary test for the evaluation of intracranial circulation for BD and its demonstrated utility over several decades, no uniform protocols include the procedure or its interpretation. As Greer and colleagues point out, catheter placement (aortic arch vs selective carotid or vertebral arteries), contrast volume, injection rate and pressure, and image acquisition rate and duration all have the potential to influence results.

The interpretation criteria of BD also lack uniformity. The American Academy of Neurology (AAN) standards published in 1995 state that, with conventional angiography, BD consists of "No intracerebral filling at the level of the carotid bifurcation or circle of Willis" (http://www. onelegacy.org/docs/AmericanAcademyNeurology_Brain DeathDetermination_1995.pdf). However, whether "carotid" refers to the common carotid artery or to the internal carotid artery is not specified. In the 2010 update, this criterion was changed to "No intracerebral filling should be detected at the level of entry of the carotid or vertebral artery to the skull" (https://www.aan.com/Guidelines/ Home/GetGuidelineContent/816).

In their letter, Drs. Greer, Wijdicks, and Kaufmann state, "Conventional angiography evaluates for arrest of flow at the point of entry of all arteries intradurally, both in the anterior and posterior circulations." We agree that the terminology for defining intracranial flow can be problematic. Moreover, on the basis of CTA studies, including our own, a requirement of "no intracranial flow" might be overly stringent because basal arterial segment filling ("stasis filling") may be present in the absence of functional cerebral circulation. This finding, when present, is likely due to the increased sensitivity of CTA to small amounts of intravascular contrast and/or the relative delay of CTA images in BD (about 60 seconds postinjection) compared with the delay of cerebral angiography images (about 10 20 seconds postinjection). Although we did evaluate our patients for flow in the vertebral and basilar arteries using the 4-point scale of Frampas et al., ${ }^{1}$ we believe that doing so was justified because their large multicenter series validated the scale as a method for diagnosing the absence of an intact intracranial circulation.

Regarding the claim of Greer et al. that our study included only 7 patients who met BD criteria, this point disregards the gold standard against which we compared our CTA results. As we stated previously, the gold standard diagnosis of BD that we used was clinical assessment alone or clinical assessment plus conventional cerebral angiography in the presence of confounding factors (most often, a positive urine toxicology result). Our stated numbers therefore hold true to the gold standard, and our subsequent statistical analyses are correct.

The larger issue underlying the entire topic of BD testing is whether we, as physicians, actually know what we are assessing. The 1981 Uniform Determination of Death
Act (http://www.uniformlaws.org/shared/docs/determina tion\%20of\%20death/udda80.pdf) states that "An individual who has sustained either 1) irreversible cessation of circulatory and respiratory functions, or 2) irreversible cessation of all functions of the entire brain, including the brain stem, is dead. A determination of death must be made in accordance with accepted medical standards." The courts and the legal system may define the standard of $\mathrm{BD}$, but we in the medical community are left to determine the exact criteria to use to identify patients who meet that standard. We therefore add to these short criteria the need to confirm the permanency of cessation of entire brain and brainstem function.

Most people believe that the AAN 2010 criteria are the standard, but there is tremendous variability in the interpretation of these criteria. ${ }^{2}$ More importantly, some courts have questioned the criteria, including the Supreme Court of the State of Nevada, which recently declared, "We are not convinced that the AAN guidelines are considered the accepted medical standard" that can adequately measure all functions of an entire brain. ${ }^{4}$ This controversy is not surprising in light of the number of inconsistencies between the AAN criteria of 1995 and the AAN criteria of 2010, with no explanation for why these changes occurred, such as minimum required body temperature $\geq 36.5^{\circ} \mathrm{C}$ versus $>36.0^{\circ} \mathrm{C}$, systolic blood pressure $\geq 90 \mathrm{~mm} \mathrm{Hg}$ versus $\geq 100 \mathrm{~mm} \mathrm{Hg}$, no formal recommendation on an acceptable period of elapsed time to verify permanency of symptoms (in either set of guidelines), variability in apnea testing (in 1995 it was acceptable to declare BD if the patient became unstable during apnea testing as long as the patient had a $\mathrm{PCO}_{2} \geq 60 \mathrm{~mm} \mathrm{Hg}$ and no respirations, yet in 2010 this same scenario required confirmatory testing), and no comment on the tremendous limitations (arguably disqualifying) of electroencephalography and transcranial Doppler ultrasonography in BD testing.

The AAN guidelines rest on an inherently incorrect assumption that by performing such clinical tests, we can diagnose death (or cessation of function) of the entire brain and brainstem; Wijdicks and Pfeifer ${ }^{5}$ demonstrated beautifully in their pathology analysis of clinically brain-dead patients that the AAN criteria do not sufficiently confirm death of the entire brain and brainstem. Furthermore, Rady and Verheijde 3 pointed out in their commentary that the AAN clinical criteria do not so much describe BD as they do a severe neurological disability, which is proven, ${ }^{5}$ in as many as $60 \%$ of cases, not to equate with biological death.

To make progress on this highly complex issue, we must redefine what $\mathrm{BD}$ means within the context of prognosis, not as a false notion of histopathological death. We must also recognize the shortcomings of the current clinical guidelines in modern-day practice, that is, that many patients experience BD with depressant drugs in their systems, severe comorbidities, disfiguring injuries that make cranial nerve testing impossible, and a myriad of other confounders. Confirmatory tests are necessary because so many patients have confounding factors present within a time frame that is both amenable to organ transplantation and compassionate to family members who are looking to their physicians for concrete information and guidance in a particularly difficult situation. We remain confident that 
our results, as well as those of others, demonstrate the acceptability of CTA as an ancillary test for BD, especially when CTA results are considered against the results of currently accepted tests, such as electroencephalography.

Michael A. Bohl, MD
C. Roger Bird, MD
Mark P. Garrett, MD
Nicholas Theodore, MD
Barrow Neurological Institute, St. Joseph's Hospital and Medical Center,
Phoenix, AZ

Phoenix, AZ

\section{References}

1. Frampas E, Videcoq M, de Kerviler E, Ricolfi F, Kuoch V, Mourey F, et al: CT angiography for brain death diagnosis. AJNR Am J Neuroradiol 30:1566-1570, 2009

2. Greer DM, Wang HH, Robinson JD, Varelas PN, Henderson GV, Wijdicks EFM: Variability of brain death policies in the United States. JAMA Neurol 73:213-218, 2016

3. Rady MY, Verheijde JL: American Academy of Neurology Guidelines and the neurologic determination of death. JAMA Neurol 73:760-761, 2016 (Letter)

4. Supreme Court of the State of Nevada: Hailu v Prime Healthcare, 68531, 2015 (http://caseinfo.nvsupremecourt.us/ document $/$ view.do?csNameID=36740\&csIID=36740\&deLin $\mathrm{kID}=530188 \&$ sireDocumentNumber $=15-34970$ ) [Accessed August 15, 2017]

5. Wijdicks EFM, Pfeifer EA: Neuropathology of brain death in the modern transplant era. Neurology 70:1234-1237, 2008

INCLUDE WHEN CITING

Published online November 24, 2017; DOI: 10.3171/2017.4.JNS17824.

CAANS 2018, except where prohibited by US copyright law

\section{Determining brain death using computed tomography angiography}

TO THE EDITOR: We read with interest the article by Garrett et al. '2 (Garrett MP, Williamson RW, Bohl MA, et al: Computed tomography angiography as a confirmatory test for the diagnosis of brain death. J Neurosurg [published online ahead of print March 17, 2017. DOI: 10.3171/2016.10.JNS161042]). We felt motivated to address the considerations below.

Recently, we published a systematic review of the role of computed tomography angiography (CTA) in the diagnosis of brain death (BD). ${ }^{1}$ In this review, according to the quality tools applied, the studies retrieved from online databases (MEDLINE, the Cochrane Library, Embase, and LILACS) were of low quality, especially because of the potential for patient selection bias. In these studies, CTA examiners were not blinded to patient clinical status, and there were no eligible controls, with the latter being the reason that CTA specificity has not been calculated. Furthermore, in many situations, the time interval between clinical testing for BD and CTA scanning was excessively long, and the CTA images were evaluated by a radiologist who knew that the clinical evaluation revealed findings that were positive for BD; consequently, there was no possibility to assess interobserver heterogeneity. The authors of 2 studies did not perform venous-phase scanning. ${ }^{1}$
Despite all limitations and statements above, our review, plus 2 other systematic reviews, ${ }^{3,6}$ found an $85 \%-87 \%$ sensitivity of CTA for detecting intracranial circulatory arrest, concluding that this technique could be applied as an ancillary test for BD diagnosis. To date, the American Academy of Neurology ${ }^{8}$ has not approved the use of CTA for $\mathrm{BD}$ diagnosis.

The study of Garrett et al.,2 despite a small control group sample $(\mathrm{n}=2)$, is the first to include eligible critically injured neurological patients who did not meet all neurological criteria for BD. This study found $100 \%$ specificity, which is of great importance, because ancillary testing should never indicate circulatory arrest in a patient in a deep coma who does not fulfill clinical neurological criteria for BD diagnosis.

The authors used the 4-point CTA scale recommended by the French Society of Neuroradiology, ${ }^{4}$ which considers CTA positive for BD in the absence of opacification of distal middle cerebral arteries (MCAs) and internal cerebral veins. Recently, the current 4-point scale has been revised, ${ }^{5}$ indicating a lack of blood flow in the deep brain drainage as the most reliable criterion to determine intracranial circulatory arrest. Nevertheless, these criteria have not been studied in cases of deep venous thrombosis, which hypothetically could preclude the use of CTA for this purpose due to the potential for false-positive results. However, false-negative results may be seen in patients with skull defects, decompressive craniectomy, or large cerebral resection, which may delay the collapse of the MCAs and allow them to remain filled with contrast. It is important to consider that cerebral circulatory arrest is linked to critical intracranial pressure; thus, all methods that assess cerebral circulation may need variable amounts of time to detect intracranial circulatory arrest.

CTA has advantages over other techniques that are currently approved for diagnosing $\mathrm{BD}$. The cost is lower and the availability is greater than those for digital subtraction angiography (DSA), scintigraphy, nuclear medicine perfusion test, and SPECT. Moreover, CTA is not operator dependent and demands a lower contrast volume, which is infused through a peripheral vein. However, there are some limitations associated with this technique that may restrict its use, such as patient transportation from the intensive care unit to the CTA unit, and renal failure, although some studies have demonstrated that venous contrast might not affect the results of kidney transplantation.?

In the study by Garrett et al., ${ }^{2}$ there is the potential for bias due to the lack of blinding in patient selection and the absence of blinding of the radiologist, who knew the patients' neurological status; all patients underwent clinical assessment for BD prior to CTA scanning. For the sample of 11 patients who did not fulfill clinical criteria for BD (1 patient had cardiac instability during apnea testing and 10 patients had CNS depressors within their systems), the comparison of CTA with DSA findings was appropriate for study purposes, although for daily practices, BD diagnosis is primarily clinical, and the ancillary test should not replace clinical evaluation; rather, they are complementary.

A diagnosis of BD will lead to withdrawal of all support and intensive care; therefore, such a determination cannot tolerate incorrect results. As Garrett et al. ${ }^{2}$ men- 
tioned, this diagnosis is essentially clinical, and ancillary tests will be applied in specific circumstances when the clinical assessment for BD cannot be completed. However, some countries around the world consider ancillary testing mandatory in all BD cases ${ }^{1}$ as a useful register for legal issues. Currently, there is ongoing research on this subject, which is registered with ClinicalTrials.gov (registration no. 12500913400000068). In this trial, comatose patients with Glasgow Coma Scale scores $\leq 5$ underwent CTA scanning and transcranial Doppler (TCD) ultrasonography. Arterial and venous images were obtained, and 2 blinded neuroradiologists analyzed CTA images; the TCD operator was also blinded. After CTA and TCD acquisition, neurological assessment was completed with efforts to allow only a short time between tests, from pupillary to apnea testing, and patients were stratified based on whether clinical criteria for BD were positive or not. Finally, the study of Garrett et al. ${ }^{2}$ is the first to be considered of higher quality on this subject. Further studies with a similar design are needed.

\section{Sergio Brasil, MD, MSc Marcelo de-Lima-Oliveira, MD, MSc Edson Bor-Seng-Shu, MD, PhD Manoel Jacobsen Teixeira, MD, PhD Hospital das Clínicas, University of São Paulo, School of Medicine, São Paulo, Brazil}

\section{References}

1. Brasil S, Bor-Seng-Shu E, de-Lima-Oliveira M, Azevedo MK, Teixeira MJ, Bernardo L, et al: Role of computed tomography angiography and perfusion tomography in diagnosing brain death: a systematic review. J Neuroradiol 43:133-140, 2016

2. Garrett MP, Williamson RW, Bohl MA, Bird CR, Theodore $\mathrm{N}$ : Computed tomography angiography as a confirmatory test for the diagnosis of brain death. J Neurosurg [epub ahead of print March 17, 2017. DOI: 10.3171/2016.10.JNS161042]

3. Kramer AH, Roberts DJ: Computed tomography angiography in the diagnosis of brain death: a systematic review and metaanalysis. Neurocrit Care 21:539-550, 2014

4. Leclerc X: [CT angiography for the diagnosis of brain death: recommendations of the French Society of Neuroradiology (SFNR).] J Neuroradiol 34:217-219, 2007 (Fr)

5. Marchand AJ, Seguin P, Malledant Y, Taleb M, Raoult H, Gauvrit JY: Revised CT angiography venous score with consideration of infratentorial circulation value for diagnosing brain death. Ann Intensive Care 6:88, 2016

6. Taylor T, Dineen RA, Gardiner DC, Buss CH, Howatson A, Pace NL: Computed tomography (CT) angiography for confirmation of the clinical diagnosis of brain death. Cochrane Database Syst Rev 3:CD009694, 2014

7. Vigneau C, Fulgencio JP, Godier A, Chalem Y, El Metaoua S, Rondeau E, et al: The use of contrast media in deceased kidney donors does not affect initial graft function or graft survival. Kidney Int 70:1149-1154, 2006

8. Wijdicks EFM, Varelas PN, Gronseth GS, Greer DM:

Evidence-based guideline update: determining brain death in adults. Report of the Quality Standards Subcommittee of the American Academy of Neurology. Neurology 74:1911-1918, 2010

\section{Disclosures}

The authors report no conflict of interest.

\section{Response}

No response was received from the authors of the original article.

\section{INCLUDE WHEN CITING}

Published online November 24, 2017; DOI: 10.3171/2017.6.JNS171335.

@AANS 2018, except where prohibited by US copyright law

\section{Function outcome related to aggressive surgery for meningioma?}

TO THE EDITOR: We read with interest the article by Gousias et al. ${ }^{1}$ (Gousias K, Schramm J, Simon M: The Simpson grading revisited: aggressive surgery and its place in modern meningioma management. J Neurosurg 125:551-560, September 2016). The authors retrospectively analyzed 901 patients with primary meningiomas. They found that more aggressive resections (Simpson grade I vs II vs III vs IV) were associated with a better, rather than a lower, postoperative performance score and that new postoperative neurological deficits or worsening preexisting ones also correlated inversely with the Simpson grade. Finally, they identified a younger age, higher preoperative performance score, and convexity tumor location-but not the degree of resection-as independent predictors of a good functional outcome. However, we believe some points require clarification.

Ouyang et al. performed a study and identified complete resection, rich blood supply, tumor adhesion, and encasement as prognostic factors for a worse postoperative Karnofsky Performance Scale (KPS) score. ${ }^{3}$ Tumor adhesion and encasement affect the degree of resection, but these factors were not included in the study conducted by Gousias et al., which resulted in different conclusions.

On the other hand, the KPS score can vary with time after surgery. ${ }^{2}$ The functional outcome in the perioperative period can be different from that in the long-term followup. Functional outcome should be analyzed by subgroup analysis according to values of the KPS score in the different periods.

In the case of multiple meningiomas, Gousias et al. analyzed only 1 meningioma per patient. But how did they select the target meningioma? Selection of the target meningioma would introduce bias, and the excluded meningiomas could affect the functional outcome. Furthermore, 58 patients were diagnosed with multiple meningiomas, and patients with a neurofibromatosis Type 2 (NF2) diagnosis should have been excluded, but the criteria for patient selection did not mention this point.

In conclusion, degree of resection as a prognostic factor for functional outcome is still controversial, and further study must be performed. Radical resection is the most important treatment for meningioma. While the risk of surgery should not be ignored by neurosurgeons and incomplete resection should be considered as an acceptable option, quality of life has surpassed other considerations.

Baoyin Shan, MM Jing Zhang, MD 
Jianguo $\mathrm{Xu}, \mathrm{MD}, \mathrm{PhD}$

West China Hospital, West China Medical School, Sichuan University, Chengdu, Sichuan, People's Republic of China

\section{References}

1. Gousias K, Schramm J, Simon M: The Simpson grading revisited: aggressive surgery and its place in modern meningioma management. J Neurosurg 125:551-560, 2016

2. Li D, Wu Z, Ren C, Hao SY, Wang L, Xiao XR, et al: Foramen magnum meningiomas: surgical results and risks predicting poor outcomes based on a modified classification. J Neurosurg 126:661-676, 2017

3. Ouyang T, Zhang N, Wang L, Li Z, Chen J: Sphenoid wing meningiomas: surgical strategies and evaluation of prognostic factors influencing clinical outcomes. Clin Neurol Neurosurg 134:85-90, 2015

\section{Disclosures}

The authors report no conflict of interest.

\section{Response}

We thank Shan et al. for their thoughtful comments on our article. They concluded that while a radical resection remains the therapy of choice for many patients with meningiomas, incomplete resections should be considered as an acceptable alternative in cases in which aggressive surgery carries increased surgical risks. Quality of life issues may be as important or even more important in meningioma surgery than aiming at a surgical cure.

We certainly agree with Shan et al. However, we could show in our study that a policy of aggressive resection is not necessarily associated with adverse functional outcomes. In our series, more radical resections (Simpson grade I vs II vs III vs IV) correlated with a better, rather than a worse, postoperative KPS score (good functional outcome defined as a KPS score of 90 or 100) in the univariate analysis. This may partially reflect the fact that our cohort included many patients with "easy to resect" tumors, such as meningiomas of the convexity, who usually demonstrate a high postoperative KPS score even after aggressive resections. Indeed, after adjustment for the meningioma site in the multivariate analysis, the degree of resection was no longer a significant predictor of the postoperative KPS score.

Hence, our data do not contradict the (in fact, somewhat conflicting) results published by Ouyang et al. ${ }^{2}$ and $\mathrm{Li}$ et al. ${ }^{1}$ In contrast to us, these authors analyzed postoperative outcomes in meningioma subgroups defined by tumor site, that is, in a series of 53 patients with sphenoid meningiomas and 185 patients with foramen magnum meningiomas, respectively. Of note, surgery for skull base meningiomas is associated with above-average surgical risks. Operations for foramen magnum tumors carried an increased risk for an adverse functional outcome in our series. We did not analyze factors such as blood supply, encasement of major neurovascular structures, or characteristics of the surgical cleavage plane for their impact on the patients' postoperative outcomes. In our view, the major impact of tumor site on such items somewhat precludes their study in a large, unselected meningioma cohort.

Shan et al. also commented on the lack of long-term outcome data. We readily accept this criticism. Indeed, we have specifically mentioned this as one of the limitations of our study. However, functional deficits tend to improve over time. Hence, one would expect long-term studies to show fewer persistent adverse effects, to identify more recurrences, ${ }^{3}$ and thus to favor aggressive surgical management. Shan and coworkers also point to the problem of including patients with multiple meningiomas (and NF2) in our analysis. We have tried to account for these issues by treating tumor multiplicity as a confounding variable statistically. Of note, only 58 of our patients $(6.4 \%)$ were diagnosed with more than 1 tumor either initially or during follow-up. Nevertheless, we have repeated our statistical analysis after excluding the latter patients. The results were essentially the same as in the overall cohort (data not shown).

Our study primarily aimed at demonstrating the oncological relevance of aggressive resections since this practice has been recently challenged. ${ }^{4}$ We did not question whether aggressive surgery could result in relevant morbidity. Rather, we feel that the relation between surgical aggressiveness and adverse outcomes depends heavily on the specifics of the various tumor sites and individual tumor characteristics. Hence, as evidenced by our data and despite the limitations of our analysis, we believe that neurosurgeons should not be deterred from pursuing aggressive meningioma resections whenever such a strategy appears reasonably safe.

\section{Konstantinos Gousias, MD, DSc University of Bonn Medical Center, Bonn, Germany University Hospital of Bochum Bergmannsheil, Bochum, Germany \\ Matthias Simon, MD University of Bonn Medical Center, Bonn, Germany Bethel Clinic, Bielefeld, Germany}

\section{References}

1. Li D, Wu Z, Ren C, Hao SY, Wang L, Xiao XR, et al: Foramen magnum meningiomas: surgical results and risks predicting poor outcomes based on a modified classification. J Neurosurg 126:661-676, 2017

2. Ouyang T, Zhang N, Wang L, Li Z, Chen J: Sphenoid wing meningiomas: surgical strategies and evaluation of prognostic factors influencing clinical outcomes. Clin Neurol Neurosurg 134:85-90, 2015

3. Pettersson-Segerlind J, Orrego A, Lönn S, Mathiesen T: Long-term 25-year follow-up of surgically treated parasagittal meningiomas. World Neurosurg 76:564-571, 2011

4. Sughrue ME, Kane AJ, Shangari G, Rutkowski MJ, McDermott MW, Berger MS, et al: The relevance of Simpson Grade I and II resection in modern neurosurgical treatment of World Health Organization Grade I meningiomas. J Neurosurg 113:1029-1035, 2010

\section{INCLUDE WHEN CITING}

Published online December 22, 2017; DOI: 10.3171/2017.6.JNS171089.

CAANS 2018, except where prohibited by US copyright law

\section{Tempering enthusiasm for new endovascular devices}

TO THE EDITOR: We read with great interest the 
recent article by Raymond et al. ${ }^{2}$ reporting on FIAT (the Flow Diversion in Intracranial Aneurysms Trial) and would like to congratulate them on their work (Raymond J, Gentric JC, Darsaut TE, et al: Flow diversion in the treatment of aneurysms: a randomized care trial and registry. $J$ Neurosurg 127:454-462, September 2017). We found this article of particular value not only because of the authors' effort but also because of the trial's implications and consequent warnings to our neurovascular community related to liberal use of flow diverter devices (FDDs). A study that supports conclusions against current tendencies should be praised.

Four years ago, we performed, in our department, a systematic review and meta-analysis of data on the use of FDDs. ${ }^{1}$ Our main conclusion was that these devices should be employed cautiously because the level of heterogeneity in the analyzed studies was too great to allow FDD placement to be considered a standard treatment for cerebral aneurysms. We recommended the use of more standardized treatments until the publication of conclusive trials, as has now happened with FIAT. Curiously, articles referencing our paper have usually misinterpreted it and have included it as a meta-analysis supporting the use of FFDs. As a matter of fact, we undertook that review alarmed by the unrestrained use of FDDs, extending far beyond the indication that they were initially approved for (the treatment of proximal internal carotid artery [ICA] aneurysms difficult to treat with standard interventions). With increasing frequency in many forums and papers, clinicians are encouraged to use FDDs for the treatment of even relatively small, simple aneurysms, including lesions distal to the ICA.

Currently, however, the situation is worsening with the continuous bombardment of information about the amazing virtues of new devices and new "eras": the FDD era, the Web era, the Medina era, and so on. The speed of appearance and introduction into medical practice of new endovascular devices is much faster than the capability of incorporating them into our practice, and we believe this situation is very dangerous. The neurovascular community should be cautious and avoid feeling overexcited immediately after the appearance of new theoretically perfect solutions. It is imperative that new regulations be adopted to control the use and abuse of unproven devices until their safe indications and technique can be adequately determined. It is good to remember that trials like ISAT only demonstrated efficacy of coiling compared to clipping and should not be interpreted as showing that any endovascular treatment is as good as or better than open surgery.

Finally, we would like again to congratulate Raymond and colleagues because their trial goes beyond their conclusions about the role of FDDs in the treatment of cerebral aneurysms. Their findings may be considered a red flag for the tendency to overestimate the virtues of new devices and techniques by comparing them against wellknown open or endovascular treatments supported by the passage of time.

Ignacio Arrese, MD, PhD

Rosario Sarabia, MD, PhD

Hospital Universitario Río Hortega, Valladolid, Spain

\section{References}

1. Arrese I, Sarabia R, Pintado R, Delgado-Rodriguez M:
Flow-diverter devices for intracranial aneurysms: systematic review and meta-analysis. Neurosurgery 73:193-200, 2013

2. Raymond J, Gentric JC, Darsaut TE, Iancu D, Chagnon M, Weill A, et al: Flow diversion in the treatment of aneurysms: a randomized care trial and registry. J Neurosurg 127:454462,2017

\section{Disclosures}

The authors report no conflict of interest.

\section{Response}

We thank Drs. Arrese and Sarabia for their much-needed support. We have not yet recruited sufficient number of patients (213 so far) in FIAT to find out when flow diverters should be used in practice. But we do hope to have shown that if innovations are to be used at all, they should be used within the context of a randomized trial. We certainly would like to see more centers collaborate in a global effort to make our practices safer and more effective.

The problem of embracing novelties without convincing evidence of superiority extends widely beyond flow diverters, as Drs. Arrese and Sarabia point out in their letter. We share with the authors the disappointment of seeing colleagues and regulatory agencies accept a case series of 100 carefully selected patients (without controls) as sufficient evidence to replace standard treatments. Although it is sad to see power and financial resources diverted away from the conduct of randomized trials, we do not think that the solution is more regulatory oversight. The solution can only be from within: more physician self-discipline. We cannot wait for others to tell us what to do. The path to the best clinical practice for all of us can only go through randomized trials designed by physicians for physicians and their patients. We continue to seek participating centers, as only a concerted international effort will yield the much sought-after answers. In the meantime, and before we gain this knowledge, we emphasize that the best management of patients is within a randomized care trial. ${ }^{1}$

Tim E. Darsaut, MD

University of Alberta, Edmonton, AB, Canada

Jean Christophe Gentric, MD

$\mathrm{CHU}$ Cavale Blanche, Brest, France

Daniela lancu, MD

University of Ottawa, The Ottawa Hospital, Ottawa, ON, Canada

Jean Raymond, MD

Centre Hospitalier de l'Université de Montréal (CHUM), Notre-Dame Hospital, Montreal, QC, Canada

\section{References}

1. Raymond J, Darsaut TE, Altman DG: Pragmatic trials can be designed as optimal medical care: principles and methods of care trials. J Clin Epidemiol 67:1150-1156, 2014

\section{INCLUDE WHEN CITING}

Published online January 5, 2018; DOI: 10.3171/2017.10.JNS172487.

CAANS 2018, except where prohibited by US copyright law 